\title{
Studien über die Wiederherstellung des Bluteiweisses sowie dessen kolloid=osmotischen Drucks unter verschiedenen Bedingungen.
}

I. Mitteilung: Versuch an hungernden Kaninchen.

\author{
Von \\ Hideo Sibuya. \\ (偨 谷 唀 雄) \\ (Aus der Medizinischen Klinik von Prof. Dr. T. Kato, \\ Tohoku-Reichsuniversität zu Sendai.)
}

Die 1906 durch Morawitz ${ }^{1)}$ erstmalig inaugurierte Plasmaphäresis, ein Verfahren, das ermöglicht aus dem kreisenden Blut, ohne jegliche Verminderung der Erythrozyten in demselben zu bedingen, möglichst grösste Eiweissmengen zu eliminieren, hat das Problem der Regeneration des Bluteiweisses ohne $Z$ weifel an das neue Light gefördert. Seither hat dieses Problem eine grosse Reihe Forscher, Ke r r, Hurwitz u. Whipple, ${ }^{2)}$ Smith, Belt u. Whipple, ${ }^{3)}$ Barker, Leiter, ${ }^{5)}$ Shelburne u. Egloff, $\left.{ }^{6}\right)$ Lepore, ${ }^{7}$ Darrow, Hopper u. Cary, ${ }^{8)}$ Dalla Volta ${ }^{9)}$ u. a. beschäftigt. Die Meinungen über den Vorgang der Wiederherstellung des Bluteiweisses gehen allerdings ziemlich weit auseinander; manche Autoren behaupten, dass bei der Regeneration des Bluteiweisses zuerst vorwiegend das Albumin zum Vorschein komme, während andere Autoren das Globulin als Erstling ansprechen. Da in keinem ihrer Experimente die Bestimmungen der

1) Morawitz, Beitr. z. chem. Physiol. u. Pathol., 1906, 7, 153.

2) Kerr, Harwitz u. Whipple, Amer. Journ. Physiol., 1918, 47, 356.

3) Smith, Belt u. Whipple, Ibid., 1920,52, 54 .

4) Barke r, Arch. Intern. Med., 1930, 45, 319.

5) Leiter, Proc. Soc. Exp. Biol. \& Med., 1930, 27, 1002 ; Arch. Intern. Med., $1931,48,1$.

6) Shelburne u. Egloff, Arch. Intern. Med., 1931, 48, 51.

7) Le p o re, Arch. Intern. Med., 1932, 50, 488.

8) Darrow, Ho pper u. Cary, Journ. Clin. Invest., 1932, 11, 683.

9) Dall a Volta, Boll. Soc. Ital. Biol. Sper., 1935, 10, 163 u. 167. 
Eiweisskörper im Blute jedoch über eine längere Zeitdauer hinaus nach Ausführung der Plasmaphäresis fortgeführt wurden, hat Fu k u ha ra ${ }^{10}$ an Kaninchen in normalen und pathologischen Zuständen für 6 Tage nach vorgenommener Plasmaphäresis den Modus der Wiederherstellung des Bluteiweisses sowie die Veränderungen des kolloid-osmotischen Drucks des Blutes (k. o. D.) verfolgt. Angesichts der Tatsache, dass der k. o. D. an sich nicht allein mit Lebensvorgängen des, Organismus aufs engste zusammenhängt, sondern, wie Starling ${ }^{11)} u$. Sör rense ${ }^{12)}$ bereits erwiesen haben, auch von der Grösse der Molekularaggregate des Bluteiweisses abhängig ist, sind den Bestimmungen des k.o.D., die nach erfolgter Plasmaphäresis fortlaufend vorgenommen werden, grosse Bedeutung zuzumessen, weil man sich auf diese Weise die Grösse der bei der Wiederherstellung des Bluteiweissbildes zum Vordergrund auftretenden Eiweissteilchen mit grösster Wahrscheinlichkeit zu mutmassen vermag.

Fukuhara ${ }^{10}$ konnte nachweisen, dass die Regeneration des Eiweisses selbst relativ rasch erfolgt und dass, weil aber am Anfang der Regeneration zuerst die Eiweissteilchen von weniger osmoaktiver, grossmolekulärer Form in grösseren Mengen vorkommen, die Wiederherstellung des k.o.D. verzögert ist. Ferner hat er gefunden, dass bei Funktionsschädigungen von Niere, Leber, Milz und Blutdrüsen die Wiederherstellung des Bluteiweisses sowie des k. o.D. ausgesprochen entstellt wird.

Indessen, weil sich die Bildung des Bluteiweisses ausserordentlich fein und kompliziert gestalten muss, bleiben eine ganze Reihe Fragen nach dem Verhalten der Eiweissregeneration unter verschiedenen Bedingungen unentschieden erübrigt, die weiterer Bearbeitung und Aufklärung bedürfen. In Erwägung dieser Sachlage habe ich einschlägige Studien unternommen, in der Absicht, weitere Beiträge zur Erfassung der Bluteiweiss- und Kolloidosmoregulation liefern zu können, indem ich bei Kaninchen, die unter verschiedene Bedingungen versetzt waren, die Plasmaphäresis vornahm und das Verhalten der danach eintretenden Wiederherstellung des Bluteiweisses sowie der Veränderungen des k. o. D. für eine längere Zeitdauer verfolgte.

In vorliegender Mitteilung sei darüber mitgeteilt, wie die Wiederherstellung des Bluteiweisses und des k.o.D. dann vor sich geht, wenn

10) Fukuhara, Tohoku Journ. Exp. Med., 1937, 30, 465.

11) Starling, Journ. Physiol., 1896, 19, 312.

12) Sörensen, C. r. Travaux Lab. Carlsberg, 1915, 12, 262. 
der Organismus im Hungerzustand der Plasmaphäresis unterworfen worden ist.

Sasaki ${ }^{13)}$ untersuchte an einzelnen Organen bei Tieren die Gewichtsabnahme durch Hunger und fand, dass sie sich in ungleichmässiger Weise verteilt, indem z.B. bei Kaninchen die Leber und die Milz gegenuiber der Abnahme des Körpergewichtes sehr rasch an Gewicht abnahmen. Ebenso auch Tana ka ${ }^{14)}$ konstatierte im Hungerversuch an Kaninchen, dass die Leber in 6 tägiger Karenz schon die Hälfte ihres ursprünglichen Gewichtes verliert. Yam a m o to ${ }^{15)}$ hat im Hungerversuch an Kaninchen den Nachweis erbracht, dass das Organeiweiss von Leber, Hirn, und Niere erhebliche qualitative Veränderungen erfährt. Es kann also nicht wundernehmen, dass an der Leber, die als das Vorratsorgan des Eiweisses eine dominierende Rolle spielt, im Hungerzustand ihr Vorratseiweiss erhebliche sowohl qualitative wie auch quantitative Veränderungen erfährt. Es lässt sich leicht vermuten, dass auch an den Muskeln, die ebenso wie die Leber als ein wichtiges eiweisshaltiges Organ gelten, ähnliche Veränderungen auftreten. Dass der Hungerzustand demgemäss beträchtliche Veränderungen auch des Bluteiweisses verursacht, ist von Blumenthal, ${ }^{16)}$ Abderhalden, Bergell u. Dörpinghaus, ${ }^{17)}$ Schoeneich, ${ }^{18}$ Morgulis u. Edwards, ${ }^{19)}$ Schelling, ${ }^{20)} \mathrm{Panum},{ }^{21)}$ Burckhardt, ${ }^{22)}$ Robertso ${ }^{23)}$ erkannt worden. Des weiteren haben neuerdings $\mathrm{Ha}$ tafuku u. Nakazawa, ${ }^{24)}$ Yamamoto, ${ }^{15)}$ an hiesiger Klinik den Beweis geführt, dass beim Hunger qualitative Veränderungen des Eiweisses mit bestimmten Veränderungen des k. o. D. des Blutes einhergehen. Es ist von Interesse, einen Einblick darin zu gewinnen, welches Verhalten das Eiweiss und der k. o. D. des Blutes aufweisen, wenn der Organismus im Hungerzustand der Plasmaphäresis unterworfen worden ist. In Hinsicht darauf wurde der vorliegende Versuch angestellt.

13) Sa saki, Jika Zasshi, 1928, 2158.

14) Tan ak a, Igaku Kenkyu, 1937, 11, 449.

15) Ya mamoto, Tohoku Journ. Exp. Med., 1938, 33, 354.

16) Blumenthal, Dtsch. med. Wschr., 1903, 437.

17) Abderhalden, Bergell a. Dörpinghaus, Ztschr. f. physiol. Chem., 1904, 41, 153.

18) S choeneich, Ztschr. f. exp. Pathol. u. Therap., 1905-6, 6, 419.

19) Morgulis u. Edwards, Amer Journ. Physiol., 1924, 68, 477.

20) Schelling, Journ. Biol. Chem., 1930, 89, 575.

21) Panum, Virchow's Arch., 1864, 29, 241.

22) Burckhardt, Arch. f. exp. Pathol. u. Pharmakol., 1883, 16, 322.

23) Roberts on, Journ. Biol. Chem., 1912-13, 13, 325.

24) Hatafuku u. Nakazawa, Tohoku Journ. Exp. Med., 1933, 21, 226. 
Versuchsmethodik: Im vorliegenden Versuch wurden $\mathrm{Ka}$ ninchen benutzt. Die Technik der Plasmaphäresis und die Methode zur Bestimmung des Eiweisses und k. o. D. waren ganz dieselben, die von $\mathrm{Fukuhara}{ }^{10}$ ) ausführlich beschrieben wurden, weshalb ihre Beschreibung hier unterlassen werden kann.

Das Prinzip der Plasmaphäresis besteht darin, dass man einem Versuchstier ca. $30 \mathrm{ccm}$ Blut pro $\mathrm{kg}$ Körpergewicht entnimmt, und dass man zugleich das einem anderen Tier entzogene Blut vom Plasma befreit, die hier zurückgebliebenen, mit physiologischer Kochsalzlösung gewaschenen Erythrozyten mit gleichem Quantum der Normosallösung wie die entzogene Plasmamenge vermischt, und die so hergestellte Mischung dem zuerst genannten Versuchstier injiziert.

Was den Hungerversuch anbelangt, habe ich anfangs nach 7 Hungertagen die Plasmaphäresis vorgenommen und dabei beabsichtigt, unter Fortsetzung des Hungerversuchs an den mehrmals entnommenen Blutproben die Bestimmungen von Hämoglobin, Eiweiss und k. o. D. auszuführen. Da aber bei derart längerem Andauern des Hungerzustandes Versuchstiere meistens bald nach Ausführung der Plasmaphäresis erlagen, wurde 5 Tage nach Beginn des Hungerversuchs die Plasmaphäresis ausgeführt und hernach 3, 6, 24 Stunden und weiterhin alle 24 Stunden die Blutproben zur Ermittelung der erforderlichen Daten entnommen; es wurden somit die Daten für 96 Stunden lang, also durch 4 Tage hindurch nach Plasmaphäresis gehoben. Weitere Bestimmungen mussten unterlassen werden, weil wegen der Erschöpfung der Versuchstiere die Blutentnahme unmöglich war.

Oben angeführte Bestimmungen wurden im absoluten Hungerversuch, wo also kein Wasse̊r gegeben wurde, vorgenommen. Nach Untersuchungen von Yamamoto ${ }^{18)}$ scheint der absolute Hungerversuch verschieden vom Hungerversuch mit Wassergabe auszufallen; es besteht nämlich dazwischen ein Unterschied in der Eiweissmobilisation. Aus Rüicksicht darauf wurden in einer anderen Versuchsreihe die Bestimmungen im Hungerversuch mit Wassergabe ausgeführt. Da in diesem Fall durch Wassergabe die Erschöpfung bei Versuchskaninchen verspätet eintritt, konnte die Plasmaphäresis nach 5 tägigem Hunger ausgeführt und das Verhalten von Eiweiss und k. o. D. bis zum Ablauf von darauf folgenden 144 Stunden, also 6 Tage hindurch verfolgt werden.

\section{Versuch an hungernden Kaninchen mit Wasserentbehrung.}

In vorliegender Versuchsreihe wurden 6 gesunde Kaninchen von 
ungefähr $2 \mathrm{~kg}$ Körpergewicht verwendet. Es wurden zuvor das Hämoglobin, Serumeiweiss und der k. o. D. des Blutes bestimmt und nachdem die Versuchstiere danach im absoluten Hungerzustand ohne Wassergabe 5 Tage lang gehalten worden waren, wurde die Plasmaphäresis nach üblicher Methode vorgenommen, und vor und nach derselben die eben genannten Daten bestimmt. Diese Versuchsresultate sind in Tab. 1 zusammengestellt.

Im übrigen wurde auch wie $\mathrm{Fukuhara}{ }^{10}$ ) bei normalen Kaninchen der Modus der nach Plasmaphäresis auftretenden Wiederherstellung des Bluteiweisses und des k. o. D. verfolgt; dabei gewonnene Er-

\section{Tabelle 1}

Bestimmungen an hungernden Kaninchen mit Wasserentbehrung.

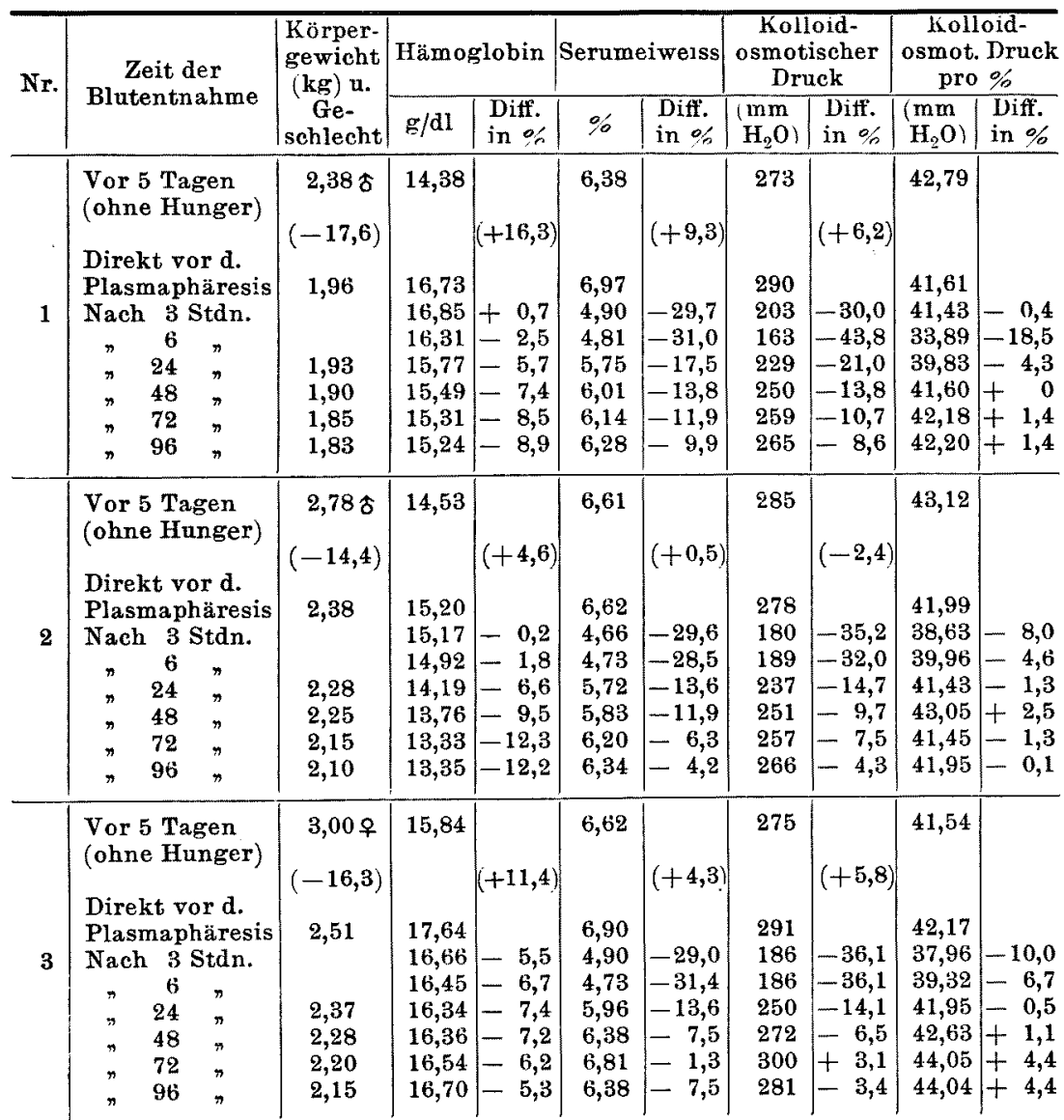




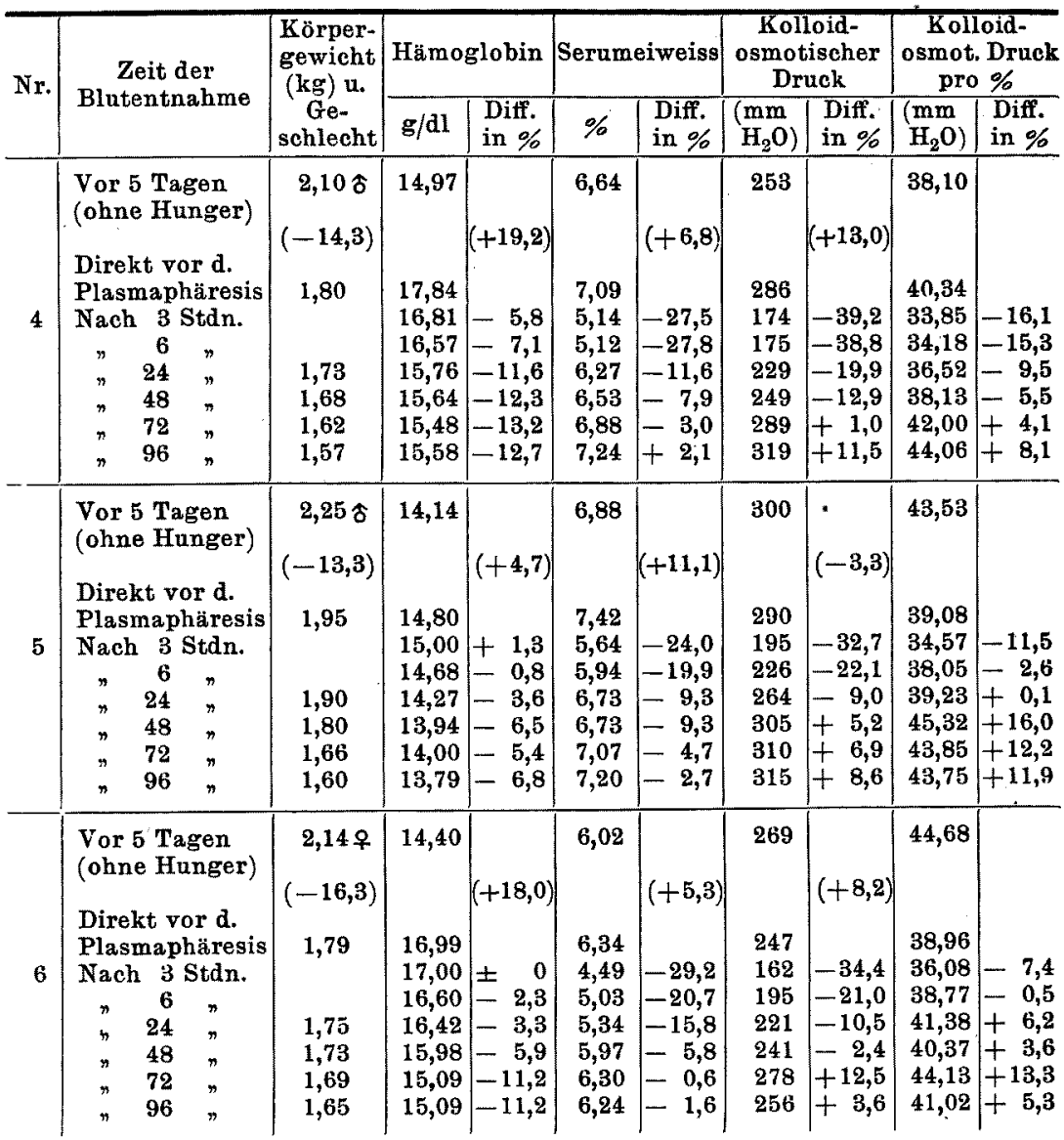

gebnisse stimmen im grossen ganzen mit Resultaten von $\mathrm{F} \mathrm{u} \mathrm{k} \mathrm{u} \mathrm{ha} \mathrm{ra}{ }^{10}$ ) überein. Ein typischer Versuchsbeispiel ist in Fig. 1 kurvenmässig angegeben.

Wenn man nun an Hand der Tab. 1 die Werte vor Beginn des Hungerversuchs mit den Werten, die unmittelbar vor Ausführung der Plasmaphäresis, nämlich am 6. Hungertage ermittelt wurden vergleicht, so ergibt sich dass das Körpergewicht in 6 Versuchen durch den 5 tägigen Hunger um 13,3-17,6\% (im Durchschnitt 15,4\%) abnahm. Hämoglobin erfuhr hierbei eine Zunahme von 4,6-19,2\% (im Durchschnitt 12.4\%), Serumeiweiss auch eine Zunahme von 0,5-11,1\% (im Durchschnitt 6,2\%). K. o.D. des Blutes nahm in 3 von 6 Versuchen (Versuch 1, 3 und 4) mehr oder weniger zu, aber in übrigen 3 Versuchen 
(Versuch 2, 5 und 6) hingegen gewissermassen ab und da der An- und Abstieg des k. o. D. mit der Eiweisszunahme nicht gleichen Schritt hielt, sank der Druck pro \% in 4 Versuchen augenscheinlich ab, während er in anderen 2 Versuchen mehr oder weniger erhöht war.

Fig. 1. Prozentuelle Veränderungen des Hämoglobins, des Bluteiweisses, des kolloid-osmotischen Drucks und des Drucks pro \% von Blut nach Plasmaphäresis bei einem normalen

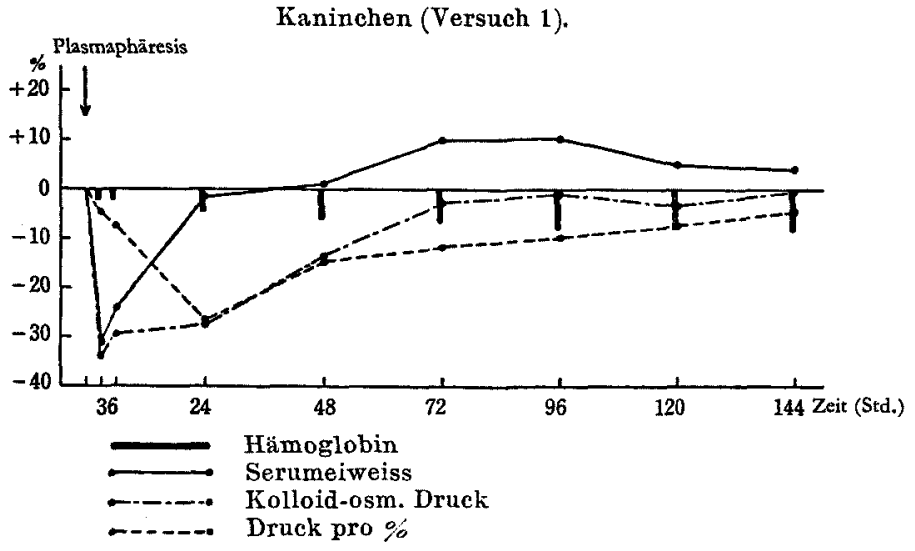

Hatafuku u. Nakazawa ${ }^{24)}$ haben bereits nachgewiesen, dass der k. o. D des Blutes sich je nach der Länge der Karenzdauer verschieden verhält, indem der k. o. D. und Druck pro \% beim kurzfristigen Hunger absinken, beim langfristigen umgekehrt ansteigen Die Diskrepanz von k. o.D. und Druck pro \% in meinem Versuch dürfte sich dadurch erklären, dass die Hungerperiode in meinem Versuch sich gerade in der Mitte zwischen von $\mathrm{Hatafuku} \mathrm{u.} \mathrm{Naka-}$ $\mathrm{zawa}^{24)}$ sog. längerer und kürzerer Karenzzeit befindet. Die Diskussion darüber kann weiter unberührt gelassen werden, weil dies mit dem Ziel meines Experiments in keinem direkten Zusammenhang steht.

Wenn nach Ablauf von 5 Hungertagen die Plasmaphäresis vorgenommen wurde, sanken Serumeiweisswerte und k. o. D.-Werte ausserordentlich ab. Die prozentuelle Abnahme des Serumeiweisses nach 3 Stunden betrug nämlich 24,0-29,7\%, noch stärker war die Abnahme von k. o. D., die sich auf 30,0-39,2\% belief. Mithin betrug die Abnahme des Drucks pro \% 0,4-16,1\%. In nach 6 Stunden entnommenen Blutproben verhielten sich das Serumeiweiss und der k. o. D. im allgemeinen nicht so sehr abweichend von Werten in eben erwähnter dreistündiger Blutportion, sie zeigten aber in einigen Fällen mehr oder minder schon eine Neigung zur Wiederherstellung. In 24 stündiger Blutprobe erfuhren das Serumeiweiss und der k. o. D. ebenfalls Zunahmen, zeigten jedoch gegenüber der Norm noch weitgehend niedrige Werte, indem 
nämlich, verglichen mit Normalwerten, das Serumeiweiss eine prozentische Abnahme von 9,3-17,5\%, der k. o.D. eine prozentische Abnahme von 9,0-21,0\% aufwies. Wenn man Abnahmen von beiden Grössen nur prozentualen Ziffern nach beurteilt, so hat es den Anschein, als ob die Wiederherstellung des Bluteiweisses derselben des k. o. D. beinahe parallel ginge. Da aber in Wirklichkeit der Grad der Abnahme des k.o.D., welche in $3 \mathrm{Std}$. nach Plasmaphäresis erfolgte, weit grösser als der der gleichzeitig aufgetretenen Abnahme des Eiweisses war, so bemerkt man, dass, nach dem Modus der Wiederherstellung beurteilt, die Wiederherstellung des k. o. D. rascher als die des Eiweisses eintrat. Um diese Verhältnisse anschaulich zu machen, ist der Verlauf in Versuch 3 als ein Beispiel dafür kurvenförmig angegeben (Fig. 2).

Fig. 2. Prozentuelle Veränderungen des Hümoglobins, des Bluteiweisses, des kolloid-osmotischen Drucks und des Drucks pro \% von Blut nach Plasmaphäresis bei einem absolut hungernden Kaninchen (Versuch 3 ).

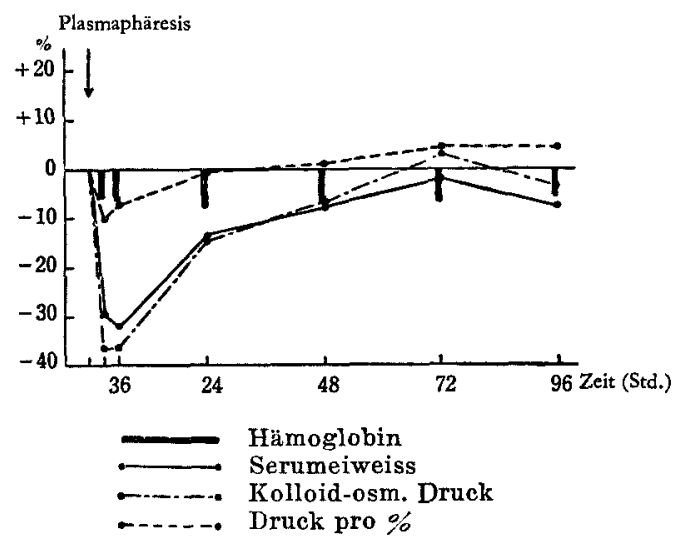

Wie aus der Fig. 1 ersichtlich, ist derartiges Verhalten unter normalen Verhältnissen nie anzutreffen. In der Norm tritt nämlich die Zunahme des Eiweisses rasch in Erscheinung, während die Wiederherstellung des k. o. D. aber recht verzögert ist, dementsprechend sinkt der Druck pro \% in dieser Periode (nach 24 Std.) auffallend ab. Da indessen im Hungerzustand in derselben Zeitspanne der k. o. D. rascher als das Eiweiss ansteigt, zeigt der Druck pro \% schon die Tendenz zur mehr oder.weniger Wiederherstellung, in manchen Versuchen(Versuch $2,3,5)$ wird hier der Anfangswert schon erreicht, in einem Versuch (Versuch 6) wird der Ursprungswert überschritten. 
In den nachfolgenden Perioden von 48,72 und 96 Std. nach Plasmaphäresis tritt die Wiederherstellung des Eiweisses im allgemeinen sehr langsam ein. Während im normalen Zustand das Serumeiweiss in diesen Perioden schon über den Anfangswert hinaus geht und öfters noch darüber in erheblichem Masse erhöht ist, zeigt das Eiweiss im Hungerzustand in 4 von 6 Versuchen nach 96 Std. noch gegenüber dem Ursprungswert niedrigere Werte. Allein die Wiederherstellung des k. o.D. geht entweder derselben des Eiweisses parallel oder überholt dieselbe, so dass der Druck pro \% in wenn auch geringem Masse, uber den Anfangswert hinaussteigt. In Versuch 1 und 2 wird um den 48 stündigen Ablauf herum der Ursprungswert um ein Geringes überschritten, in 4 Versuchen (Versuch 3, 4,5 und 6) erhöht sich der Druck pro $\%$ mit dem Zeitverlauf immer erheblicher. Derartige Erscheinung lässt sich unter normalen Verhältnissen vermissen; im normalen $\mathrm{Zu}$ stand tritt die Wiederherstellung des k. o. D. im Vergleich mit der des Eiweisses ausserordentlich verzögert ein, wobei die Wiederherstellung des Drucks pro \% dementsprechend erst nach 120 Std. nach Plasmaphäresis erfolgt.

Nach alledem lässt sich also sagen, dass im absoluten Hungerzustand die Wiederherstellung des Eiweisses erheblich verzögert ist und dass die Wiederherstellung des k. o. D. hingegen sehr schnell erfolgt, während der Druck pro \% nach Ablauf von 48 Std. den Anfangswert überschritten hat.

\section{Versuch an hungernden Kaninchen, ohne Wasserentbehrung.}

An 5 gesunden Kaninchen wurde der Hungerversuch nach dem gleichen Verfahren, wie es in vorheriger Versuchsreihe ausgeführt wurde, angestellt, hierbei wurden aber während der Karenzdauer jedem Versuchstier täglich einmal frühmorgens ca. $20 \mathrm{ccm}$ Wasser mittels Sonde in den Magen eingeführt. Nach gleicher Methode wie in vorangehender Versuchsreihe wurde 5 Tage nach Beginn des Hungerversuchs die Plasmaphäresis vorgenommen, worauf das Verhalten von Serumeiweiss und k. o. D. verfolgt wurde. Es wurde Wasser auch nach Vornahme der Plasmaphäresis einmal täglich bis zum Abschluss der Bestimmung gegeben.

Was das Körpergewicht in unmittelbar der Plasmaphäresis vorangehender Periode, d. h. am sechsten Hungertage anbelangt, verloren Versuchstiere 5,4-16,9\% (im Durchschnitt 11,6\%) ihres Ursprungs- 
gewichtes, die Abnahme war gegenüber derselben im absoluten Hunger etwas geringfügig.

Die Hämoglobineindickung war nicht so hochgradig wie im absoluten Hunger, in 3 Versuchen trat eine geringgradige Eindickung auf, während in anderen 2 Versuchen eher die Tendenz zu mässiger Verdünnung zum Vorschein kam. Serumeiweiss erfuhr entweder eine geringe Zunahme oder blieb fast unverändert. K. o. D. und Druck pro $\%$ liessen keine wesentlichen Veränderungen erkennen.

Die Ergebnisse, welche an Kaninchen in eben geschildertem $\mathrm{Zu}$ stand nach Ausführung der Plasmaphäresis gewonnen wurden, sind in Tab. 2 aufgezeichnet.

Die Abnahme von Serumeiweiss und k. o. D. in 3 Std. nach Plasmaphäresis betrug gleich 23,0-37,9\%, und 32,6-43,0\%. Es bestand also zwischen den beiden Grössen eine ähnliche Beziehung wie in vorangehender Versuchsreihe insofern, als das Absinken des k. o. D. grös-

\section{Tabelle 2.}

Bestimmungen an hungernden Kaninchen ohne Wasserentbehrung.

\begin{tabular}{|c|c|c|c|c|c|c|c|c|c|c|}
\hline \multirow[t]{2}{*}{ Nr. } & \multirow{2}{*}{$\begin{array}{c}\text { Zeit der } \\
\text { Blutentnahme }\end{array}$} & \multirow{2}{*}{$\begin{array}{c}\text { Körper- } \\
\text { gewjeht } \\
\text { (kg) u. } \\
\text { Ge- } \\
\text { schlecht }\end{array}$} & \multicolumn{2}{|c|}{ Hämoglobin } & \multicolumn{2}{|c|}{ Serumeiweiss } & \multicolumn{2}{|c|}{\begin{tabular}{c|} 
Rolloid- \\
osmotischer \\
Druck
\end{tabular}} & \multicolumn{2}{|c|}{$\begin{array}{c}\text { Kolloid- } \\
\text { osmot. Druck } \\
\text { pro \% }\end{array}$} \\
\hline & & & $\mathrm{g} / \mathrm{dl}$ & \begin{tabular}{|l|} 
Diff. \\
in $\%$
\end{tabular} & $\%$ & \begin{tabular}{|l|} 
Diff. \\
in $\%$
\end{tabular} & $\begin{array}{l}(\mathrm{mm} \\
\left.\mathrm{H}_{2} \mathrm{O}\right)\end{array}$ & $\begin{array}{l}\text { Diff. } \\
\text { in } \%\end{array}$ & $\stackrel{(\mathrm{mm}}{\left.\mathrm{H}_{2} \mathrm{O}\right)}$ & $\begin{array}{l}\text { Diff. } \\
\text { in \% }\end{array}$ \\
\hline \multirow{11}{*}{1} & \multirow{4}{*}{$\begin{array}{l}\text { Vor } 5 \text { Tagen } \\
\text { (ohne Hunger) } \\
\text { Direkt vor d. } \\
\text { Plasmaphäresis } \\
\text { Nach } 3 \text { Stdn. }\end{array}$} & 2,07 ชิ & 15,27 & & 6,03 & & 287 & & 47,59 & \\
\hline & & $(-16,9)$ & & $(+6,6)$ & & $(+7,6)$ & & $(+1,0)$ & & \\
\hline & & 1,72 & 16,28 & & 6,49 & & 290 & & 44,68 & \\
\hline & & & 15,61 & $-4,1$ & 4,53 & $-30,2$ & 176 & $-39,3$ & 38,85 & $-13,0$ \\
\hline & 6 & & 15,56 & $-4,4$ & 4,77 & $-26,5$ & 217 & $-25,2$ & 45,49 & $+1,8$ \\
\hline & $\Rightarrow \quad 24$ & 1,60 & 15,53 & $-4,6$ & 6,05 & $-6,8$ & 292 & $+0,7$ & 48,26 & $+8,0$ \\
\hline & $\Rightarrow \quad 48$ & 1,52 & 15,44 & $-\mathbf{5 , 1}$ & 6,08 & $-\mathbf{6 , 3}$ & 256 & $-11,7$ & 42,10 & $-5,8$ \\
\hline & 72 & 1,45 & 14,97 & $-8,0$ & 6,32 & $-2,6$ & 272 & $-6,2$ & 43,04 & $-3,7$ \\
\hline & 96 & 1,49 & 13,61 & $-16,4$ & 6,01 & $-7,4$ & 268 & $-7,6$ & 44,59 & $-0,2$ \\
\hline & $\Rightarrow 120$ & 1,44 & 13,33 & $-18,1$ & 6,38 & $-1,7$ & 272 & $-6,2$ & 42,63 & $-4,6$ \\
\hline & $\Rightarrow 144$ & 1,38 & 13,24 & $-18,7$ & 6,14 & $-5,4$ & 272 & $-6,2$ & 44,30 & $-0,8$ \\
\hline \multirow{11}{*}{2} & \multirow{2}{*}{$\begin{array}{l}\text { Vor } 5 \text { Tagen } \\
\text { (ohne Hunger) }\end{array}$} & 2,02 우 & 15,44 & & 6,98 & & 319 & & 45,70 & \\
\hline & & $(-11,4)$ & & $(+10,8)$ & & $(+7,4)$ & & $(+3,4)$ & & \\
\hline & \multirow{2}{*}{$\begin{array}{l}\text { Direkt vor } d . \\
\text { Plasmaphäresis } \\
\text { Nach } 3 \text { Stdn. }\end{array}$} & 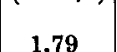 & 17. & & 7,50 & & 330 & & 44,00 & \\
\hline & & & 17,14 & $+0,2$ & 4,66 & $-37,9$ & 188 & $-43,0$ & 40,34 & $-8,3$ \\
\hline & 6 & & 16,78 & $-1,9$ & 4,92 & $-34,4$ & 191 & $-42,1$ & 38,82 & $-11,8$ \\
\hline & 24 & 1,75 & 16,63 & $-2,8$ & 5,81 & $-22,5$ & 272 & $-17,6$ & 46,81 & $+6,4$ \\
\hline & 48 & 1,6 & 16,60 & $-3,0$ & 6,32 & $-15,7$ & 300 & $-9,1$ & 47,47 & $+7,9$ \\
\hline & $" 72$ & 1,61 & 15,49 & $-9,5$ & 7,05 & $-6,0$ & 304 & $-7,9$ & 43,12 & $-2,0$ \\
\hline & $7 \quad 96$ & 1,57 & 14,55 & $-1,4,9$ & 7,11 & $-5,2$ & 282 & $-14,5$ & 39,66 & $-9,9$ \\
\hline & $\pi 120$ & 1,5 & 14,64 & $-14,4$ & 6,98 & $-6,9$ & 275 & $-16,7$ & 39,40 & $-10,4$ \\
\hline & $" 144$ & 1,54 & 14,24 & $-16,8$ & 6,64 & $-11,5$ & 270 & $-18,2$ & 40,66 & $-7,6$ \\
\hline
\end{tabular}




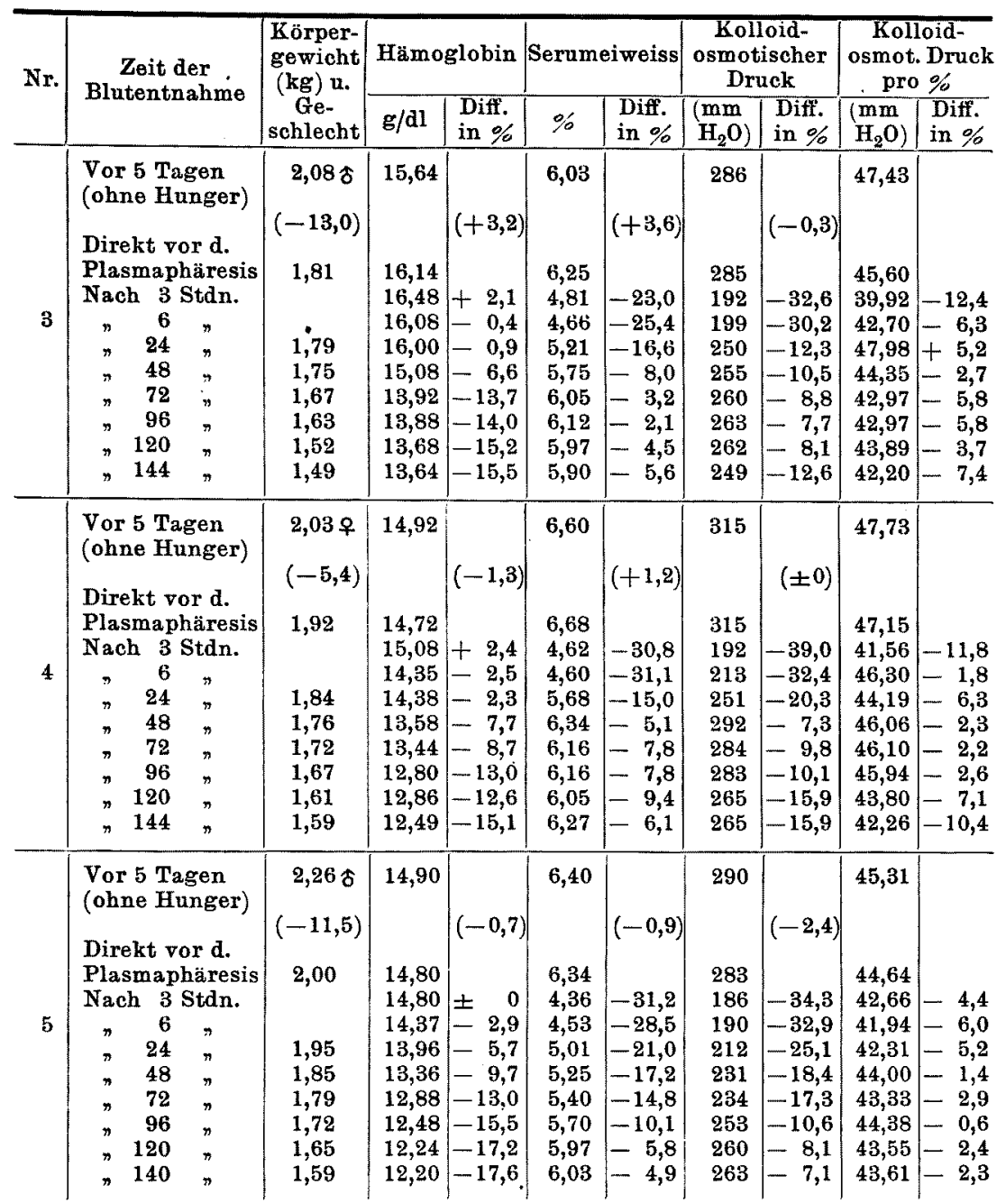

ser als die Eiweissabnahme war. Mithin war auch der Druck pro \% herabgesetzt. Nach 6 Std. erfuhr das Serumeiweiss in Versuch 3 und 4 eine weitere Abnahme, während in Versuch 1, 2 und 5 schon die Neigung zur Annäherung an das frühere Niveau angetroffen wurde. Da indessen der k. o. D. in dieser Periode schon mehr oder minder Erhöhung, also Wiederherstellung aufwies, zeigte der Druck pro \%, der nach 3 Stunden einmal herabgesetzt worden war, hier in 3 von 5 Versuchen erhebliche Steigerung. Vor allem aber in Versuch 1 stieg der Druck pro \% bereits über den Anfangswert hinaus. 
Was das Verhalten des Eiweisses und des k. o. D. nach 24 stündigem Ablauf nach Plasmaphäresis anbetrifft, zeigte das Eiweiss in dieser Periode die Tendenz zur Wiederherstellung, war jedoch weit entfernt von dem Ursprungswert. Nur in einem Fall (Versuch 1) trat es gewissermassen an den Anfangswert heran. Im Gegensatz zum Eiweiss wurde der k. o. D. sehr prompt wiederhergestellt, in 2 von 5 Versuchen (Versuch 4 und 5) hielt er mit der Erholung des Eiweisses beinahe gleichen Schritt, in 3 Versuchen (Versuch 1, 2 und 3) stellte sich er weitgehend rascher als das Eiweiss wieder her, wodurch der Druck pro \% schon über den Anfangswert hinausstieg. Um diese Beziehung zu veranschaulichen, ist die Daten des Versuchs 3 als ein typisches Beispiel kurvenförmig dargestellt (Fig. 3). Nach 48 Std. zeigte das Eiweiss die

Fig. 3. Prozentuelle Veränderungen des Hämoglobins, des Bluteiweisses, des kolloid-osmotischen Drucks und des Drucks pro \% von Blut nach Plasmaphäresis bei einem hungernden Kaninchen ohne Wasserentbehrung (Versuch 3).

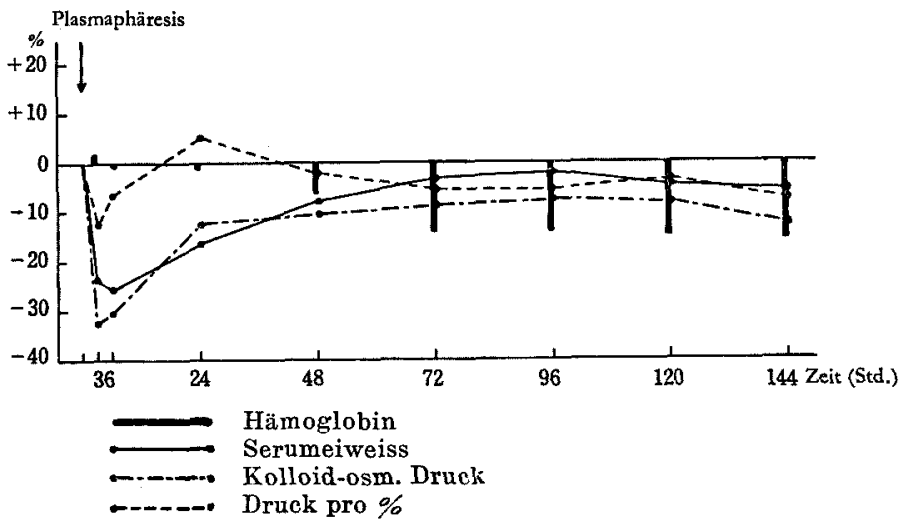

Neigung zu weiterer, wenn auch langsamer Wiederherstellung, die gegenüber der Norm allerdings sehr verzögert war. K. o. D., der bis zum Ablauf von 24 Std. rasch an den Anfangswert herangetreten war, näherte sich nach $48 \mathrm{Std}$. langsam demselben, so dass seine Wiederherstellung entweder parallel mit derselben des Eiweisses ging oder noch langsamer als diese erfolgte. Mithin erfuhr der Druck pro \% nach 48 Std. mitunter eher eine Erniedrigung. Nach 72 Std. waren das Eiweiss und der k. o. D. an der Wiederherstellung gehindert, oder anscheinend eher herabgesetzt.

Im ganzen genommen lässt sich also sagen, dass, während die Wiederherstellung des Serumeiweisses sich von derselben im absoluten 
Hunger nicht so abweichend verhält, die Wiederherstellung des k.o.D. bei regelmässiger Wasserdarreichung anfangs sehr prompt erfolgt, später (nach 48 Std.) aber so gut wie sistiert.

\section{Besprechung.'}

Während in der Norm sich das Bluteiweiss derart prompt erholt, dass es schon 24 Std. oder 48 Std. nach Ausführung der Plasmaphäresis zum Anfangswert zurückkommt, oder sogar denselben weit überschreitet, wird beim Hunger, wie die vorliegenden Versuche gezeigt haben, gleichviel, ob Wasser gegeben wird oder nicht, seine Wiederherstellung ausserordentlich verzögert. Im Gegensatz zum Eiweiss kommt der k. o.D., dessen Wiederherstellung im normalen Zustand äusserst langsam erfolgt und deshalb derselben des Eiweisses weitgehend nachsteht, im Hungerversuch beträchtlich geschwind zum früheren Niveau zurück. Und im absoluten Hungerversuch geht die Wiederherstellung des k.o.D. mehr schnell in späteren Perioden als in früheren vonstatten und übertrifft infolgedessen im allgemeinen die Wiederherstellung des Eiweisses, so dass der Druck pro \% in späteren Perioden in der Mehrzahl der Fälle über den Ursprungswert weitgehend hinaussteigt. Hingegen im Hungerversuch, wo eine bestimmte Menge Wasser täglich verabreicht wird, erfolgt die Wiederherstellung des k. o. D. eine Zeitlang nach Ausführung der Plasmaphäresis noch schneller als im absoluten Hunger, so dass der Druck pro \% in den meisten Fällen schon 6 Std. oder 24 Std. später eine Erhöhung aufweist oder sogar den Anfangswert überschreitet. In späteren Perioden vollzieht sich die Wiederherstellung des k. o. D. jedoch nicht so prompt, wie am Anfang, sondern es hat den Anschein, als ob in den meisten Fällen gleichsam eine stationäre Phase eintritt.

Von vornherein liegt es auf der Hand, dass wenn aus dem strömenden Blut das Eiweiss durch Plasmaphäresis entnommen worden ist, der Organismus zum Ersatz des verlorenen Eiweisses das Depoteiweiss mobilisiert. Die Tatsache, dass im Hunger Eiweissdepots vermindert werden, ist auch aus Abnahme des Körpergewichts und aus bereits eingangs geschilderten Bestimmungen von Tanaka ${ }^{14)}$ und Sasa $\mathrm{ki}^{13}$ zu ersehen. Die Untersuchungen dennoch, die sich mit qualitativen Veränderungen des Eiweisses im Hunger befassen, liegen meines Wissens sehr wenig vor; in dieser Richtung sind in jüngster Zeit von Yamamoto ${ }^{15)}$ an hiesiger Klinik Versuche durchgeführt worden, in denen er unter Berücksichtigung des Verhaltens des Rest-N und des 
k. o. D. die Veränderungen des Gewebseiweisses verfolgte. Nach Yama moto ${ }^{15)}$ gehen im Hungerzustand bei Leber, Hirn und Niere die Spaltungsprozesse des Gewebseiweisses gesteigert vor sich, und es kommt zur Vermehrung des Rest- $\mathrm{N}$, wobei die dabei entstandenen kleinen osmoaktiven Eiweissteilchen ins Blut abgegeben werden sollen. Jedenfalls erscheint es selbstverständlich, dass, wiewohl Eiweissvorratsorgane nach Plasmaphäresis bestrebt sind, ihr Depoteiweiss ins Blut zu mobilisieren und dadurch den Eiweissverlust zu ersetzen, aus Mangel an Vorratseiweiss, der schon durch den Einfluss des Hungers bestanden hat, eine vollkommene Wiederherstellung des Eiweissgehaltes zur Norm unmöglich bewerkstelligt wird, gleichgültig, ob dabei Wasser zugeführt wird oder nicht. Andererseits aber ergibt sich aus Versuchen von Fukuhara, ${ }^{10}$ dass der Organismus im normalen Zustand auf eine ziemlich längere Zeitdauer nach Plasmaphäresis das Eiweiss von grösserer Molekularform ins Blut abgibt und dass er erst nach quantitativ vollkommener Wiederherstellung des Eiweisses das Eiweiss von kleinerer Form ins Blut mobilisiert und somit die Regulation des k. o. D. des Blutes vornimmt. Im Zusammenhang damit ist höchst interessant die gegensätzliche Tatsache, dass im Hungerzustand. die Wiederherstellung des k. o. D. sich gegenüber der Norm weitgehend schnell vollzieht und zumal diese prompte Wiederherstellung des k.o.D. offensichtlich darauf beruht, dass Vorratsorgane für das Eiweiss von vornherein kleine osmoaktive Eiweissteilchen mobilisieren. Dies ist. meines Erachtens dahin zu deuten, dass das Vorratseiweiss, wie auch Yamamoto ${ }^{15)}$ nachgewiesen hat, durch den Einfluss des Hungers schon gespalten und in kleine Eiweissteilchen umgewandelt war oder bei seiner Mobilisation geschwind zu kleiner Form gespalten und ins. Blut abgegeben wird.

Im Hunger mit Wasserzufuhr vollzieht sich der Spaltungsprozess. in Eiweissdepotorganen durch Wirkung des Wassers in raschem Tempo, wobei das betreffende Eiweiss nicht allein hinreichend gespalten werden kann, sondern auch durch Wasser mit Leichtigkeit ins Blut abtransportiert wird, wodurch die Wiederherstellung des k. o. D. nach - Plasmaphäresis äusserst geschwind erfolgt. Dies steht in Übereinstimmung mit dem Befund von Yamamoto, ${ }^{15}$ dass bei Wasserzufuhr im Hunger der Rest- $\mathrm{N}$ und die Eiweissteilchen der Gewebe leichter ins Blut übergehen. Der Grund hierfür, warum in vorliegendem Hungerversuch nach 24 stündigem Ablauf nach Plasmaphäresis die Wiederherstellung des k. o. D. sistiert, ist aller Wahrscheinlichkeit nach darin zu suchen, dass, bei gesteigerter Spaltung und Mobilistation des De- 
poteiweisses, infolge des völligen Ausbleibens des Eiweissersatzes von aussen der Eiweissmangel sich schnell geltend macht.

\section{Schluss.}

1. Wenn bei hungernden Kaninchen die Plasmaphäresis ausgeführt worden ist, so ist im Blute die Wiederherstellung des Eiweisses gegenüber der Norm ausserordentlich verlangsamt, wohingegen die Erholung des kolloid-osmotischen Drucks rascher als in der Norm vonstatten geht.

Im Hungerzustand, wo aber eine bestimmte Menge Wassertäglich verabreicht wird, findet die Wiederherstellung des kolloid-osmotischen Drucks bis zum Ablauf von ungefähr 24 Stunden nach Plasmaphäresis mehr geschwind als bei absoluter Karenz statt, die nachfolgende Wiederherstellung ist jedoch verzögert.

2. Aus obigen Ergebnissen kann erschlossen werden, dass bei hungernden Kaninchen nach der Plasmaphäresis zuvörderst ins Blut mobilisiertes Eiweiss im Gegensatz zur Norm, aus kleinmolekulären Eiweissteilchen zusammengesetzt ist, d. h. zur Albumingruppe gehört. 